STRUCTURAL SCIENCE CRYSTAL ENGINEERING MATERIALS

ISSN 2052-5206

Received 8 October 2020

Accepted 10 January 2021

Edited by C. M. Reddy, IISER Kolkata, India

Keywords: hexagonal boron nitride; $\mathrm{AB}$ stacking; high-resolution transmission electron microscopy; simulation.

Supporting information: this article has supporting information at journals.iucr.org/b

\section{AB-stacked nanosheet-based hexagonal boron nitride}

\author{
Jae-Kap Lee, ${ }^{\mathrm{a} *}$ Jin-Gyu Kim, ${ }^{\mathrm{b}}$ K. P. S. S. Hembram, ${ }^{\mathrm{a}}$ Seunggun $\mathrm{Yu}^{\mathrm{c}}$ and \\ Sang-Gil Lee ${ }^{b}$
}

\begin{abstract}
${ }^{a}$ Center for Opto-Electronic Materials and Devices, Korea Institute of Science and Technology, Seoul, 02792, South Korea, ${ }^{\mathbf{b}}$ Division of Electron Microscopic Research, Korea Basic Science Institute, Daejeon, 34133, Republic of Korea, and 'Insulation Materials Research Center, Korea Electrotechnology Research Institute, Changwon, 51543, Republic of Korea. *Correspondence e-mail: jklee@kist.re.kr
\end{abstract}

Hexagonal boron nitride ( $h$-BN) has been generally interpreted as having an AA stacking sequence. Evidence is presented in this article indicating that typical commercial $h$-BN platelets $(\sim 10-500 \mathrm{~nm}$ in thickness) exhibit stacks of parallel nanosheets $(\sim 10 \mathrm{~nm}$ in thickness) predominantly in the $\mathrm{AB}$ sequence. The AB-stacked nanosheet occurs as a metastable phase of $h$-BN resulting from the preferred texture and lateral growth of armchair (110) planes. It appears as an independent nanosheet or unit for $h$-BN platelets. The analysis is supported by simulation of thin AB films (2-20 layers), which explains the unique X-ray diffraction pattern of $h$-BN. With this analysis and the role of pressure in commercial high-pressure high-temperature sintering (driving nucleation and parallelizing the in-plane crystalline growth of the nuclei), a growth mechanism is proposed for 2D $h$-BN (on a substrate) as 'substrate-induced 2D growth', where the substrate plays the role of pressure.

\section{Introduction}

Boron nitride $(\mathrm{BN})$ is comparable with graphite in its (layered) structure, as well as with respect to its physical properties (Wang et al., 2017). The structures of its allotropes, i.e. soft 'hexagonal' $(h-\mathrm{BN})$ and hard 'cubic' $(c-\mathrm{BN})$, are comparable with those of graphite and diamond, respectively. The specific stacking structure of $h$-BN layers has not been clarified. It was reported initially to have an $\mathrm{AB}$ stacking sequence (Hassel, 1926), similar to Bernal AB graphite (Bernal, 1924). In 1950, Pease (1950) proposed AA stacking based on the calculated X-ray diffraction pattern (XRD) intensity, and this has generally been accepted as the structure of $h$-BN (Wang et al., 2017; Pease, 1952; Topsakal et al., 2009; Constantinescu et al., 2013; Gilbert et al., 2018; Alem et al., 2009; Warner et al., 2010). The analysis pointing to AA stacking is mostly based on energy calculations, where AA is more stable by approximately tens of meV compared to AB (Constantinescu et al., 2013; Gilbert et al., 2018; Liu et al., 2003). The energy difference can lead the layers to slide even at room temperature by agitation. Liu et al. (2003) expected that real $h$-BN may have a mixture of $\mathrm{AA}$ and $\mathrm{AB}$ stacking based on the small energy difference of $\sim 0.012 \mathrm{meV}$.

Due to the presence of two elements, i.e. $\mathrm{B}$ and $\mathrm{N}, h-\mathrm{BN}$ has two structural family groups of $\mathrm{AB}$ and $\mathrm{AA}$, such as $\mathrm{AB}^{\prime}(\mathrm{B}$ over B), $\mathrm{A}^{\prime} \mathrm{B}(\mathrm{N}$ over $\mathrm{N}), \mathrm{AB}(\mathrm{B}$ over $\mathrm{N})$ and $\mathrm{AA}$ (B over B), and $\mathrm{AA}^{\prime}$ (B over $\mathrm{N}$ ) (Constantinescu et al., 2013; Gilbert et al., 2018). It is accepted that $\mathrm{AA}^{\prime}$ and $\mathrm{AB}^{\prime}$ are the minimum 
energy configurations in each group (Constantinescu et al., 2013; Gilbert et al., 2018); thus, $\mathrm{AA}$ and $\mathrm{AB}$ here refer to $\mathrm{AA}^{\prime}$ and $\mathrm{AB}^{\prime}$, respectively. Here, we also notice that the $\mathrm{AA}^{\prime}$ sequence of $h$-BN is not related to the $\mathrm{AA}^{\prime}$ sequence of graphite (Lee et al., 2008, 2016). We also expect that the similarity of the X-ray diffraction (XRD) patterns for samples of $h$ - $\mathrm{BN}$ with that for $\mathrm{AA}^{\prime}\left(2 \theta=40-50^{\circ}\right)$ is one reason for the analysis of the structure of $\mathrm{BN}$ as $\mathrm{AA}^{\prime}$ (like Pease's analysis). Commercial $h$-BN reveals a unique XRD pattern (Yuan et al., 2014; Li et al., 2011; Huang et al., 2000; Zhang et al., 2017; Matović et al., 2016), where the (100) peak at $2 \theta=41.5^{\circ}$ is stronger than the (101) peak at $2 \theta=43.7^{\circ}$. Such relative intensity of the (100) peak with repsect to the (101) peak (which is a reverse of $\mathrm{AB}$ graphite) is observable for bulk $\mathrm{AA}^{\prime}$ $h$-BN (Fig. S1 in the supporting information), while the interplanar spacing of $h$-BN (3.34 $\AA$ ) is close to the value of $3.35 \AA$ for AB graphite (Bernal, 1924; Lee et al., 2008).

With the recent focus on two-dimensional (2D) materials inspired by graphene (Novoselov et al., 2004), understanding the structure of $h$-BN is vital to tune the band gap (Dai et al., 2014; Ribeiro \& Peres, 2011), as well as to design 2D $h$-BNbased heterostructures for electronic applications (Wang et al., 2017; Behura et al., 2015). Here we show that, unlike the conventional view, $h$-BN appears predominantly as an $\mathrm{AB}$ sequence, based on a series of simulations [energy, XRD and high-resolution transmission electron microscope (HRTEM)], as well as XRD and HRTEM analyses of commercial samples, and our study is consistent with the diverse data on the structure of $h$-BN reported in the literature.

\section{Experimental}

We analyzed commercial $h$-BN (3M Technical Ceramics, Germany), revealing the typical platelet shape with dimensions of micrometres. The samples were analyzed using an $\mathrm{X}$-ray diffractometer (PANalytical X'pert Pro) with a $\mathrm{Cu} \mathrm{K \alpha}$
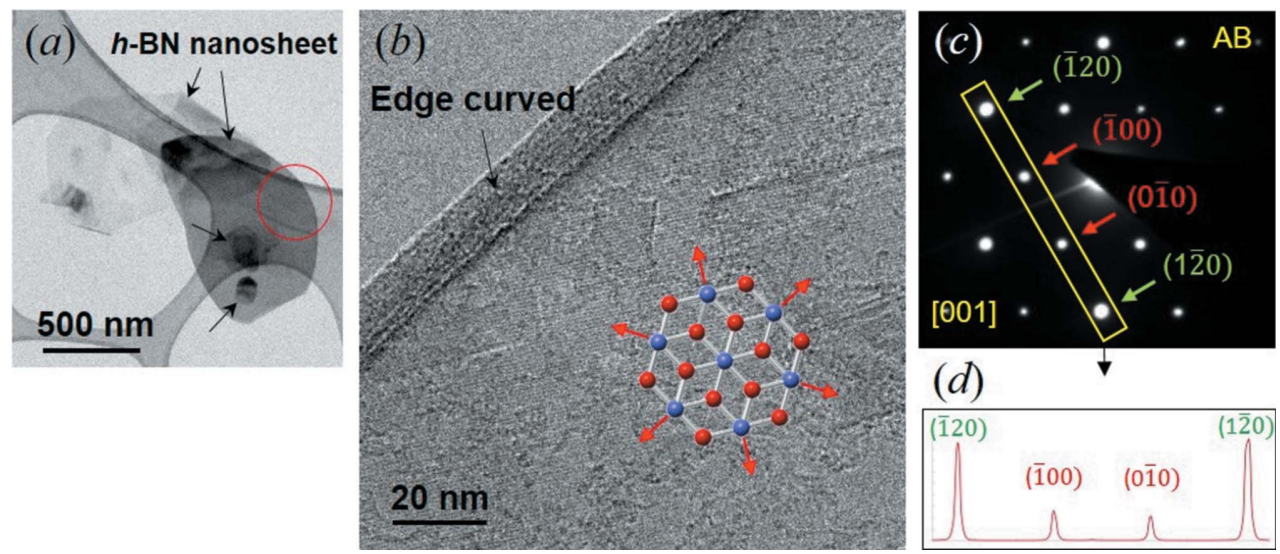

Figure 2

HRTEM images of $h$-BN nanosheets and SAED pattern analysis for a nanosheet. (a) A low magnification TEM image revealing several nanosheets (arrow) which appear to be overlapped. The red circle indicates the selected area of $\sim 400 \mathrm{~nm}$ in diameter where the ED pattern was acquired. (b) A HRTEM image of a nanosheet where the thickness is measured to be approximately $10 \mathrm{~nm}$ and an end is curved. The schematic in (b) depicts the texture growth of an $\mathrm{AB} h$-BN nucleus. (c) A SAED pattern of the nanosheet. $(d)$ An intensity profile of the spots in the rectangle in part $(c)$. The intensity ratio of $\{120\}$ to $\{100\}$ spots is 3.3 .
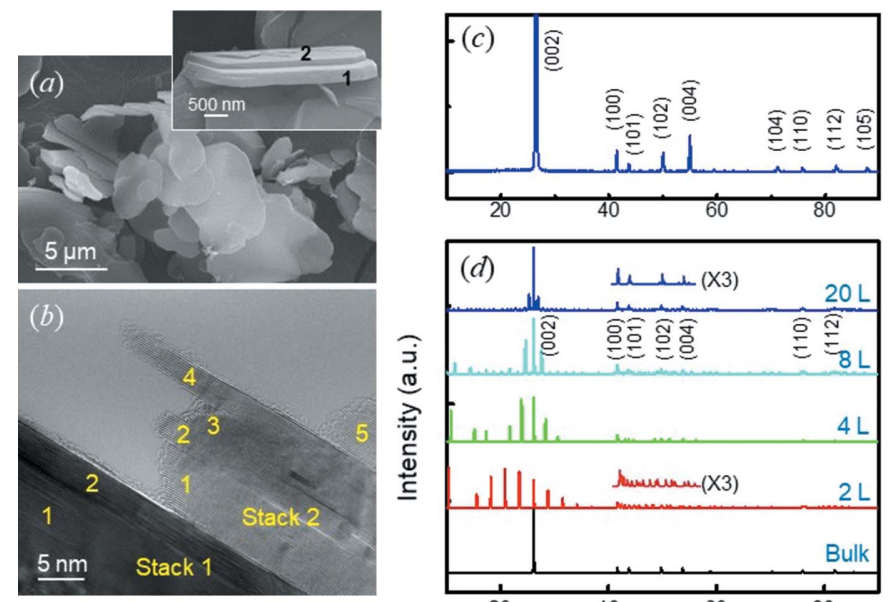

Figure 1

Analysis of $h$-BN samples. (a) A SEM image revealing the typical platelet shapes of commercial $h$-BN. The inset shows the overlap of two platelets (1 and 2), which reveals traces of sublayers (Fig. S2 in the supporting information). (b) A HRTEM image revealing stacks 1 and 2, corresponding to the two platelets shown in part $(a)$, which are expected to be composed of at least two and five nanosheets, respectively. (c) An XRD pattern measured for the $h$-BN samples. (d) Simulated XRD patterns for the $\mathrm{AB} h$-BN bulk and thin films with a different number of BN layers.

source, a scanning electron microscope (SEM) (Inspect F50, FEI) and two TEMs, i.e. Cs-corrected TEM (Libra 200 HT Mc, Carl Zeiss) and TitanTM80-300 (FEI). The selected area for the electron diffraction pattern (SAED) was acquired from a single (unstacked) BN sheet of approximately $10 \mathrm{~nm}$ in thickness (Figs. 1 and 2) to minimize the dynamical scattering effect. We used CrystalMaker (Palmer, 2014) to build atomic structures of $h$-BN for HRTEM and electron diffraction (ED) simulations, which were calculated using JEMS (CIME EPFL). Digital micrograph (Gatan Inc.) software was used to analyze the HRTEM images and their fast Fourier transform (FFT) patterns. In order to determine the suitable 1:1 combination of $\mathrm{B}$ and $\mathrm{N}$, we scanned a wide range of their spectra. We considered the homogeneous distribution of $\mathrm{B}$ and $\mathrm{N}$ in a unit cell. The bilayer structure is considered by various stacking sequences. The suitable structure is obtained by observing the stability of various structures. The simulations were carried out by first-principles calculations implemented in Quantum Espresso (Giannozzi et al., 2009). Ultra-soft pseudopotentials were used to represent interaction between ionic cores and valence electrons (Vanderbilt, 1990). 
Generalized gradient approximation was used for the exchange correlation energy of electrons (Perdew et al., 1996). Plane wave basis with an energy cut-off of $40 \mathrm{Ry}$ was used with a suitable mesh of grids (Monkhorst \& Pack, 1976; Methfessel \& Paxton, 1989). XRD patterns of all the structures were generated by the FULLPROF suite to provide a comparison with the experimental data (Rodríguez-Carvajal, 2001).

\section{Results}

\subsection{Structure analysis of $h$-BN platelets}

Fig. 1(a) shows the SEM image of commercial $h$-BN, synthesized by a high-pressure and high-temperature (HPHT) sintering process. The materials reveal the typical platelet shape where the dimensions vary widely in thickness (approximately hundreds of $\mathrm{nm}$ ) and diameter $(\sim 10 \mu \mathrm{m})$ (see also Fig. S2 in the supporting information). The samples also reveal the typical XRD pattern for $h$-BN (Fig. 1c), where the relative intensity of the (100) peak at $2 \theta=41.5^{\circ}$ and of the (101) peak at $2 \theta=43.7^{\circ}$ [where the (100) peak is stronger than the (101) peak] (Yuan et al., 2014; Li et al., 2011; Huang et al., 2000; Zhang et al., 2017; Matović et al., 2016). Such relative intensity is observable in the simulated pattern for $\mathrm{AA}^{\prime} \mathrm{BN}$ (Fig. S1 in the supporting information). Our energy calculations show that $\mathrm{AB}^{\prime}$ and $\mathrm{AA}^{\prime}$ are stable phases in each structure group, and $\mathrm{AA}^{\prime}$ is the stable phase of $h$-BN (Fig. S3 in the supporting information). The results are consistent with those reported by others (Constantinescu et al., 2013; Gilbert et al., 2018; Liu et al., 2003), where the energy differences are in the range of tens of meV. Hereafter, we refer to $\mathrm{AA}^{\prime}$ and $\mathrm{AB}^{\prime}$ simply as $\mathrm{AA}$ and $\mathrm{AB}$, respectively.

An SAED pattern, measured from a sheet of approximately $10 \mathrm{~nm}$ in thickness (Fig. 2a), is shown in Fig. 2(b), where the intensity of the $\{120\}$ spots are much stronger than those of the $\{100\}$ spots. The intensity ratio of the $\{120\}$ and $\{100\}$ spots measured from the intensity profile (Fig. $2 d$ ) was 3.3 , which is similar to that $(2.8)$ of the simulation for the $\mathrm{AB} h-\mathrm{BN}$ structure (Fig. $3 a^{\prime}$ ). The analysis demonstrates that the nanosheet is of an $\mathrm{AB}$ single-crystalline nature (Figs. $3 a$ and $\left.3 a^{\prime}\right)$. The samples ( $\sim 10 \mathrm{~nm}$ in thickness) measured here reveal evidence of $\mathrm{AB} h$-BN (Fig. S4 in the supporting information). We could not identify SAED evidence for AA (Fig. $3 d$ ). A SAED pattern for $\mathrm{AB} h$-BN was reported by Gilbert et al. (2018), who deposited $h$-BN on iron foil by the chemical vapour deposition (CVD) method. On the other hand, the revelation of the edge of the sheet (Fig. $2 b$ ) is due to the nature of the $2 \mathrm{D}$ structures where the ends appear as curved (Lee et al., 2017).

The presence of $\mathrm{AB} h$-BN is also evident in the crosssectional HRTEM images shown in Fig. 4( $\left.a^{\prime}\right)$ (see also Fig. S5 in the supporting information). White dots, due to each set of two atoms ( $\mathrm{B}$ and $\mathrm{N}$ ) on a cross-sectional HRTEM image for $\mathrm{AB} h$-BN, form a 'diagonal lattice' with an angle of $\sim 35^{\circ}$, due to the vertical lines angled by $\sim 70^{\circ}$ (exactly $67-75^{\circ}$; Fig. $3 c$ ) with repsect to the horizontal direction. This explains the hexagonal fast Fourier transform (FFT) pattern for $\mathrm{AB} h-\mathrm{BN}$ (see inset in Fig. $4 a^{\prime}$ ). Such a unique cross-sectional HRTEM morphology for $\mathrm{AB}$ was reported previously, and separately, by Tonkikh et al. (2016) and Sutter et al. (2013). Tonkikh et al. (2016) interpreted the lattices as AB, while Sutter et al. (2013) interpreted them as $\mathrm{ABC}$ rhombohedral $\mathrm{BN}(r-\mathrm{BN})$.

On the other hand, the sample shown in Fig. 4(b), is analyzed as an overlap of nanosheets. Indeed, many platelet $h$-BN samples reveal evidence of stacks comprising plural sheets of thickness $\sim 10 \mathrm{~nm}$ (Fig. 1b). With the presence of the independent nanosheet (Fig. 2), the stack of nanosheets indicates that the nanosheet is a unit of a typical $h$-BN platelet. This shows that the samples should be analyzed as a nanosheet, i.e. a textured thin structure (Lee et al., 2016).
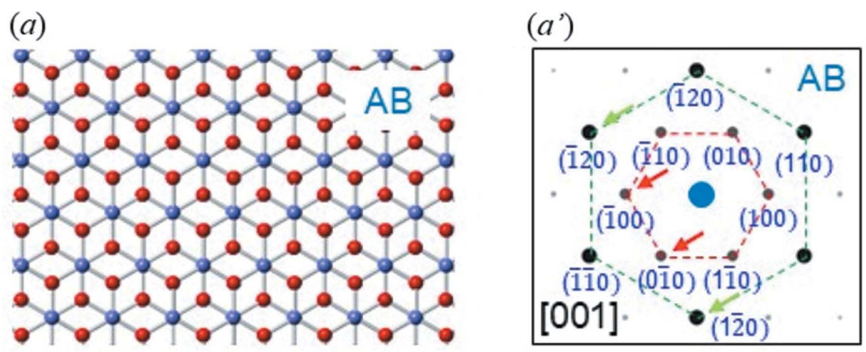

(b)

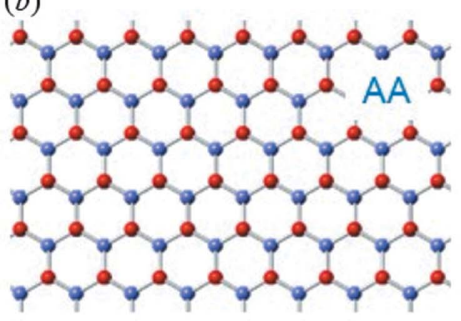

(c) $\mathrm{AB}$

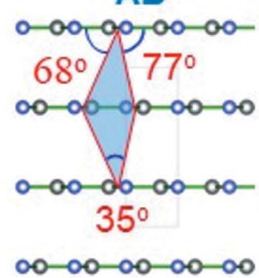

(d)

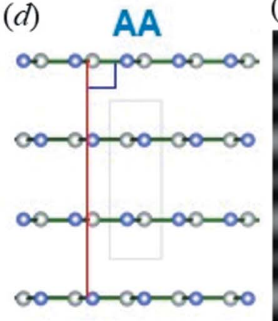

$\left(d^{\prime}\right)$

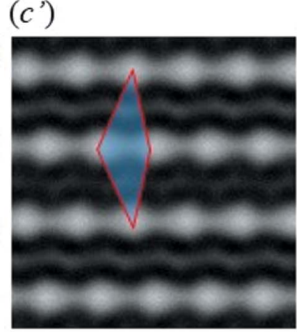

$\left(b^{\prime}\right)$
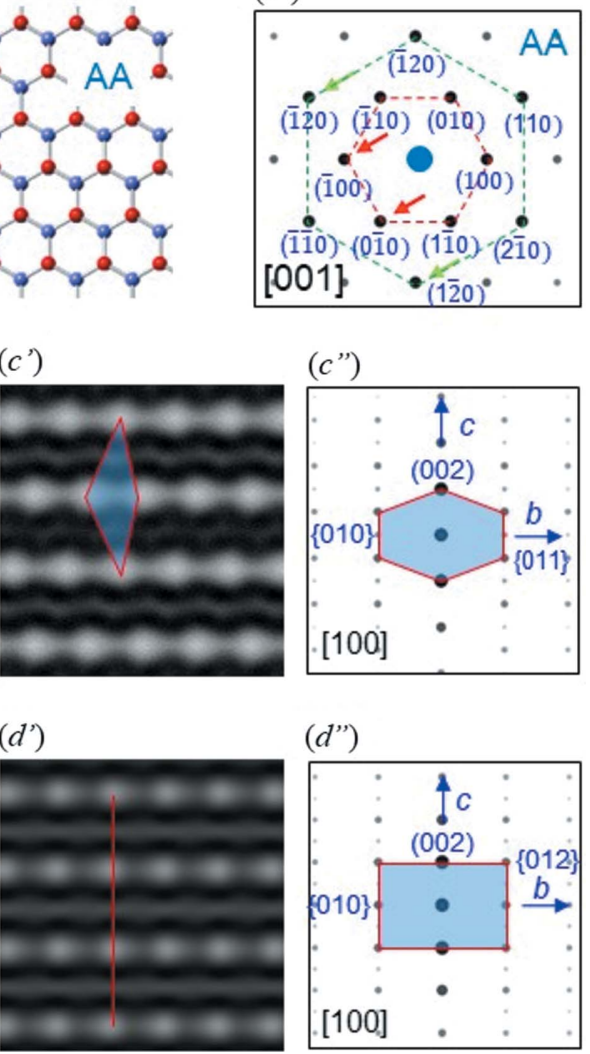

$\left(d^{\prime \prime}\right)$

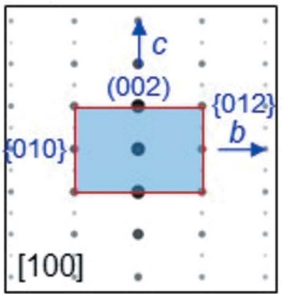

$\infty-\infty-\infty-\infty-\infty$

Figure 3

Simulated ED patterns. $(a) /(b)$ Models for plane view AB and AA $h$-BN. $\left(a^{\prime}\right) /\left(b^{\prime}\right) \mathrm{ED}$ patterns for $\mathrm{AB}$ and $\mathrm{AA} h$-BN, where the intensity ratios of the $\{120\}$ and $\{100\}$ spots are 2.8 and 0.95 , respectively. $(c) /(d)$ Models for the cross-sectional view of $\mathrm{AB}$ and $\mathrm{AA} h$-BN. $\left(c^{\prime}\right) /\left(d^{\prime}\right)$ Simulated HRTEM images for $\mathrm{AB}$ and AA $h$-BN. $\left(c^{\prime \prime}\right) /\left(d^{\prime \prime}\right)$ Simulated ED patterns for $\mathrm{AB}$ and $\mathrm{AA} h$-BN. $\mathrm{AB}$ reveals a hexagonal pattern $\left(c^{\prime \prime}\right)$, while $\mathrm{AA}$ reveals a rectangular pattern $\left(d^{\prime \prime}\right)$. 
Simulated XRD patterns for thin AB $h$-BN, shown in Fig. $1(d)$, indicate that the (101) peak becomes weaker with an increasing number of layers, resulting in the unique relative intensity of $h$-BN (Fig. 1c) (Yuan et al., 2014; Li et al., 2011; Huang et al., 2000; Zhang et al., 2017; Matović et al., 2016), while textured AA $h$-BN reveals a rather strong (101) peak (Fig. S1 in the supporting information). The data indicate that the unique XRD pattern for $h$-BN (Fig. 1c) is evidence of the $\mathrm{AB}$ nanosheet structure. The appearance of clear ED spots (Fig. 2b) and FFT (see inset in Fig. 4a) indicates that the nanosheets are of a well-developed single-crystalline nature.

\subsection{Growth mechanism of $\boldsymbol{h}$-BN}

Using the HRTEM and XRD data, we depict a formation mechanism for the $h$-BN nanosheet (Fig. 5). Due to the small energy difference, $\mathrm{AA}$ and $\mathrm{AB}$ nuclei can be formed at the initial stage of the synthesis (Fig. 5a). Their growth is dominated by the armchair (011) plane with a higher surface energy of $5.5 \mathrm{~J} \mathrm{~m}^{-2}$ compared with $4.8 \mathrm{~J} \mathrm{~m}^{-2}$ for the zigzag (100) plane (the values are for graphite) (Abrahamson, 1973). This causes the preferred $\langle 110\rangle$ texture growth of six directions on the nuclei (red arrow in Fig. 5a). With the texture growth, many (local) lateral growths (also driven by $\langle 110\rangle$ ) of the armchair (100) planes (green arrow in Fig. $5 b$ ) result in the
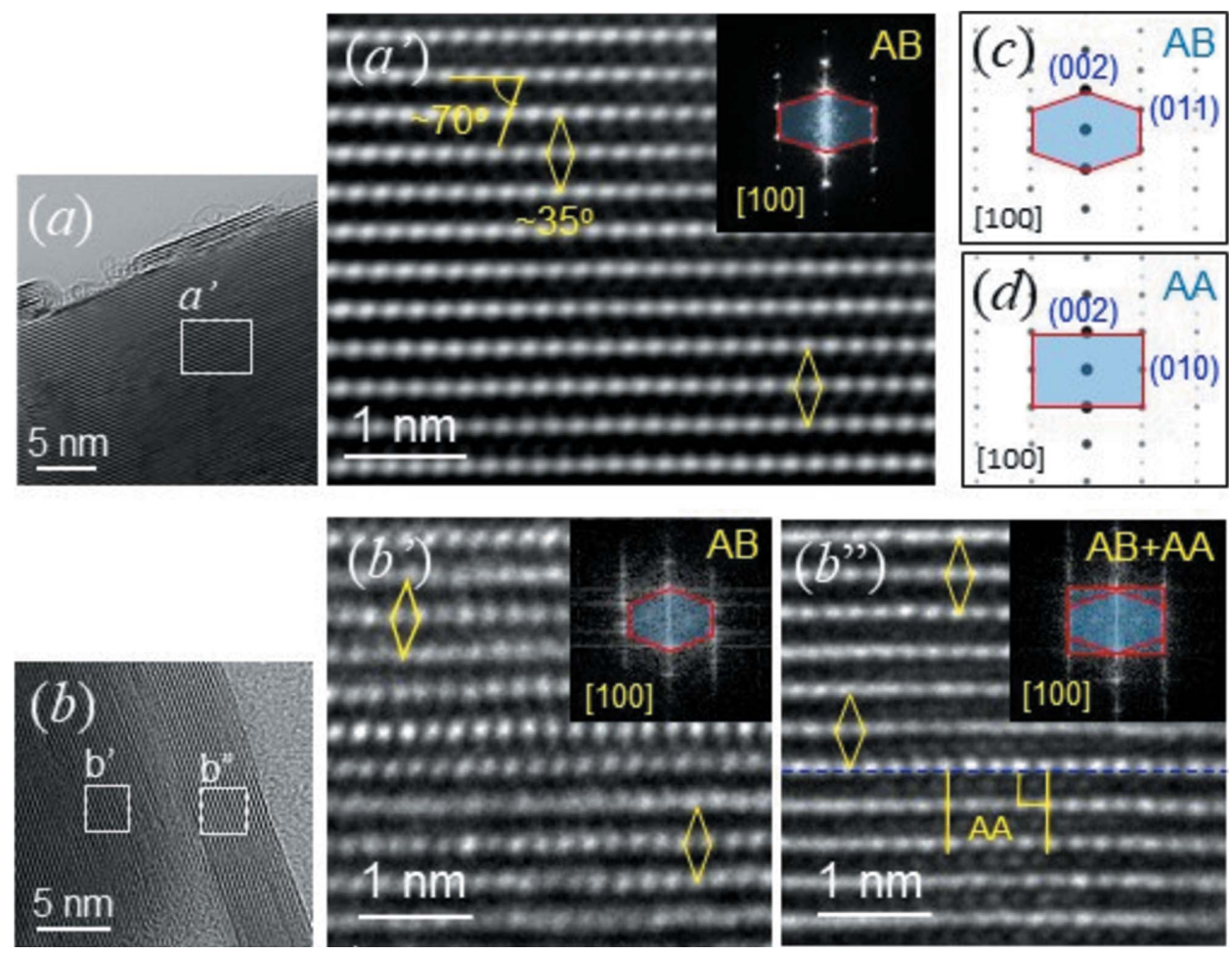

Figure 4

HRTEM images for $h$-BN samples. (a) A HRTEM image of a $h$-BN sample. ( $\left.a^{\prime}\right)$ Atomically resolved TEM image revealing the diagonal lattices of $\mathrm{AB} h$-BN. (b) A HRTEM image of a $h$-BN sample. $\left(b^{\prime}\right) /\left(b^{\prime \prime}\right)$ Atomically resolved TEM image for the rectangles in part $(b)$, revealing the diagonal lattices for $\mathrm{AB} h$-BN and the diagonal lattices for $\mathrm{AB} h$-BN. The insets in parts $\left(a^{\prime}\right),\left(b^{\prime}\right)$ and $\left(b^{\prime \prime}\right)$ indicate the FFT patterns obtained from each morphology. (c) Simulated ED pattern for AB $h$-BN. (d) Simulated ED pattern for AA $h$-BN.

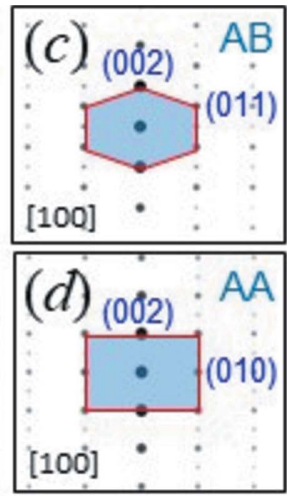

formation of the circular 2D nanosheet structure (Fig. $5 b$ ), which explains the single-crystalline sheet shown in Fig. 2.

We infer that the crystalline lateral growth of the nanosheet (Fig. 5b) occurs collectively, i.e. in the unit of the (110) plane. Here, the (110) plane of the $\mathrm{AB}$ structure, i.e. (110) $\mathrm{AB}$ with a relatively uniform distribution of the atoms, can be effective in terms of the planar growth, compared with $(110)_{\mathrm{AA}}$, where atoms are localized (Fig. $5 c$ ). We attribute the dominant existence of $\mathrm{AB}$ stacking to the relatively uniform distribution of the atoms in (110) $)_{\mathrm{AB}}$, leading to the collective growth of $(110)_{\mathrm{AB}}$. In contrast, the growth of $(110)_{\mathrm{AA}}$ may occur at the unit of the localized atoms (red dots in Fig. 5c), preventing its collective growth. We also expect that, in terms of planar growth, the zigzag edges of the (100) planes of $\mathrm{AA}$ or $\mathrm{AB}$ $h$-BN (Figs. $5 a$ and $5 b$ ) may be less effective due to the uneven arrangement of the atoms. These infer that less stable $\mathrm{AB}$ appears typically as a metastable phase of $h$-BN. The analysis is supported by the in-plane HRTEM evidence of Li et al. (2011) for $\mathrm{AB}$ nanosheets (peeled from $h$-BN platelets), revealing the diagonal lattices unique to $\mathrm{AB}$ stacking (Lee et al., 2016).

We paid attention to pressure in the commercial HPHT sintering process, which drives the nucleation of $h$-BN sheets. Under uniaxial pressure, vertical or angular growth of nuclei (against the direction of the applied pressure) might be prohibited. Such pressure-induced 2D (PI-2D) growth (Fig. 5d) parallelizes the crystalline growth of the armchair (110) planes of the $h$-BN nuclei in the vessel of the system. This explains the laminated structure of the platelets, i.e. the in-plane stack of nanosheets shown in Figs. 1(b) and $4(d)$; a typical $h$-BN platelet is approximately hundreds of $\mathrm{nm}$ thick (Fig. 1a). Here, the interface between the nanosheets can be disordered, i.e. twisted, resulting in relatively weak bonding.

The cleavage of commercial $h$ BN samples (along the basal plane of approximately hundreds of $\mathrm{nm}$ thick $h$-BN platelets into $\sim 10 \mathrm{~nm}$ thick nanosheets) with one-hour ball milling reported by Huang $e t$ al. (2000) supports our PI-2D growth model, although these authors explained the laminated decomposition as being due to Frank dislocation. This indicates that commercial $h$-BN is typically composed of nanosheets of $\sim 10 \mathrm{~nm}$ thickness $\quad(\sim 30 \quad B N$ layers). The thickness of the $h$-BN sheets may be determined at the nucleation stage, depending on the driving force (pressure and 


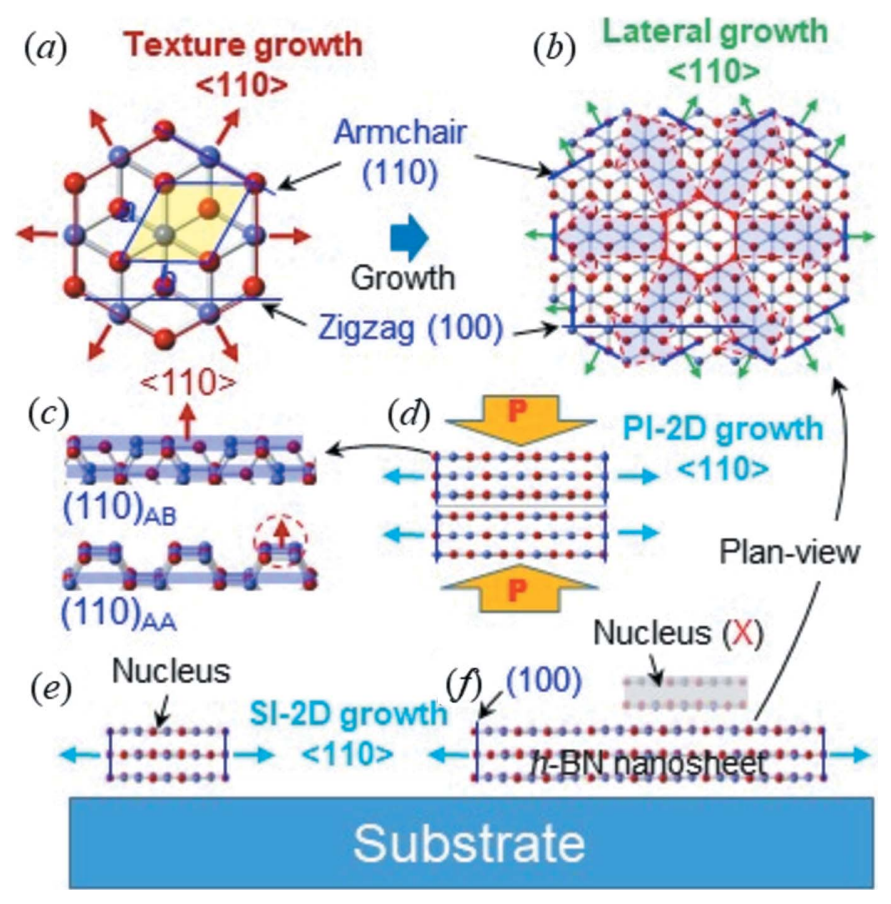

Figure 5

Growth mechanism of $h$-BN. (a) Explanation for the texture growth (red arrow) of an $\mathrm{AB}$ nucleus. (b) Explanation for the lateral growth (green arrow) of an $A B$ nanosheet. (c) Schematic showing the (110) $)_{A B}$ and $(110)_{\mathrm{AA}}$ planes for three $\mathrm{BN}$ layers [translucent blue planes indicate $(110)_{\mathrm{AB}}$ or $\left.(110)_{\mathrm{AA}}\right]$. The dashed circle indicates the localized growth of $(110)_{\mathrm{AA}}$ due to its localized arrangement of atoms. (d) Schematic explaining the PI-2D growth model of $h$-BN platelets in HPHT sintering. ' $\mathrm{P}$ ' in big arrows indicates pressure. (e) Schematic explaining the SI-2D growth model of $h$-BN on a substrate. ' $\mathrm{X}$ ' indicates the impossibility of the secondary nucleation onto the nanosheet.

temperature) applied to the system regardless of the sequence being AA or AB.

Our growth model for $h$-BN nanosheets, based on crystalline and PI-2D growth, can be extended to the 2D growth of $h$ BN on a substrate via CVD (Gilbert et al., 2018; Behura et al., 2015; Li et al., 2019) and physical vapour deposition (PVD) (Tonkikh et al., 2016; Sutter et al., 2013) approaches. Indeed, CVD and PVD grown $h$-BN reveals a nanosheet structure of $\sim 5 \mathrm{~nm}$ in thickness or a faceted (triangular or hexagonal) morphology (Gilbert et al., 2018; Li et al., 2019). The latter can be explained by Wulff construction.

Thus, we propose a 'substrate-induced 2D (SI-2D) model' for the 2D $h$-BN growth on a substrate (Fig. $5 d$ ). Here, the substrate plays the role of uniaxial pressure in the commercial HPHT process, driving heterogeneous nucleation, as well as guiding the 2D growth of the nuclei on a substrate with the unique crystalline growth of the armchair (110) planes. This SI-2D model supplements the previously suggested $2 \mathrm{D}$ growth models of $h$-BN (or graphene), including the layer-by-layer growth reported in the literature (Sutter et al., 2013; Khan et al., 2017). We expect that layer-by-layer growth or secondary nucleation onto $h$-BN nanosheets (Khan et al., 2017) may not be active in reality because both need 'collective' (001) planar growth along the $c$ axis and 'coherent' nucleation onto the inplane of the nanosheet, respectively. Due to the stability of the basal plane $\left(0.11 \mathrm{~J} \mathrm{~m}^{-2}\right)$, which is not competitive with the prismatic planes (4.8-5.5 $\mathrm{J} \mathrm{m}^{-2}$ in) (Abrahamson, 1973; Pierson, 1993), layer-by-layer growth or secondary nucleation may not occur in the 2D growth of $h$-BN. The analysis infers that the thickness of $\mathrm{BN}$ films in the CVD or PVD processes needs to be controlled at the stage of nucleation. We also infer that, in the SI-2D model, the number of BN layers (thickness) of nuclei (which grow laterally) may be affected by CVD or PVD conditions (pressure, temperature, etc.), rather than the condition of the substrate.

\subsection{Co-existence of $A B$ and $A A$ stacking}

On the other hand, we could observe HRTEM morphological evidence for AA (vertical line lattice; Fig. 3d), which appears with $\mathrm{AB}$ in a nanosheet (Fig. $\left.4 b^{\prime \prime}\right)$. Such co-existence of $\mathrm{AA}$ and $\mathrm{AB}$, reported from mechanically milled commercial platelet samples (Huang et al., 2000), confirms the results calculated by Liu et al. (2003). An independent AA nanosheet was included in the PVD samples of Sutter et al. (2013), together with another AB nanosheet. Thus, the appearance of AA is expected to be due to its crystalline growth (during sintering) and/or sliding of $\mathrm{BN}$ layers in preformed $\mathrm{AB}$ to form stable AA by agitation (after synthesis). For the latter case, the sliding of BN layers may depend on the state of a nanosheet, such as end curvature (Fig. 2b), the presence of defects (dislocations or stacking faults) (Fig. S5 in the supporting information), as well as external factors, such as mechanical milling (Huang et al., 2000) and temperature. The diffused FFT patterns shown in Figs. $4\left(b^{\prime}\right)$ and $4\left(b^{\prime \prime}\right)$ indicate that the BN layers are under strain. We attribute the strain to sliding of the BN layers to accommodate energy.

Our XRD analysis based on the simulations, providing general structural information, also supports our analysis of the structure of $h$-BN samples to be predominantly AB. The XRD simulations indicate that bilayer $h$-BN can reveal additional peaks in the range $2 \theta=42-60^{\circ}$ (Fig. $1 d$ ) due to (enlarged) relaxation of their upmost layers. This may explain the appearance of the unexpected XRD peaks at $2 \theta=42.6$ and $45.6^{\circ}$ reported by Yuan et al. (2014), who explained them as being due to $r$-BN. Indeed, their sample revealed a few $h$-BN layers, including mono- and bilayers, supporting our analysis of the XRD signal.

\section{Summary}

We reinterpreted the structure of $h$ - $\mathrm{BN}$ in terms of crystalline growth. Commercial $h$-BN platelets are composed of nanosheets in an $\mathrm{AB}$ stacking, resulting from the crystalline (texture and lateral) growth of the armchair (110) planes, as well as the PI-2D growth of AB nuclei. Stable AA stacking can appear locally. We propose a growth model for $2 \mathrm{D} h$-BN in the CVD or PVD approaches to the crystalline growth on a substrate, leading to the SI-2D growth of BN nuclei. Our results, establishing the growth and resulting structure of $h$-BN, may provide a method of preparing an ideal 2D platform for electronics comparable with graphene structures. 


\section{Acknowledgements}

The simulations were carried out by the Server farm supercomputing facility at KIST.

\section{Funding information}

The following funding is acknowledged: Korea Institute of Science and Technology (award No. 2E30432); Korea Basic Science Institute Institutional Programs (award No. D110400).

\section{References}

Abrahamson, J. (1973). Carbon, 11, 337-362.

Alem, N., Erni, R., Kisielowski, C., Rossell, M. D., Gannett, W. \& Zettl, A. (2009). Phys. Rev. B, 80, 155425.

Behura, S., Nguyen, P., Che, S., Debbarma, R. \& Berry, V. (2015). J. Am. Chem. Soc. 137, 13060-13065.

Bernal, J. D. (1924). Proc. R. Soc. London Ser. A, 106, 749-773.

Constantinescu, G., Kuc, A. \& Heine, T. (2013). Phys. Rev. Lett. 111, 036104.

Dai, S., et al. (2014). Science, 343, 1125-1129.

Giannozzi, P., et al. (2009). J. Phys. Condens. Matter, 21, 395502.

Gilbert, S. M., Pham, T., Dogan, M., Oh, S., Shevitski, B., Schumm, G., Liu, S., Ercius, P., Aloni, S., Cohen, M. L. \& Zettl, A. (2018). https:// arxiv.org/pdf/1810.04814.pdf.

Hassel, O. (1926). Nor. Geol. Tidsskr. 9, 258.

Huang, J. Y., Yasuda, H. \& Mori, H. (2000). J. Am. Ceram. Soc. 83, 403-409.

Khan, M. H., Liu, H. K., Sun, X., Yamauchi, Y., Bando, Y., Golberg, D. \& Huang, Z. (2017). Mater. Today, 20, 611-628.

Lee, J.-K., Hembram, K. P. S. S., Park, Y., Lee, S.-G., Kim, J.-G., Lee, W. \& Moon, D. J. (2017). J. Phys. Chem. Lett. 8, 2597-2601.

Lee, J.-K., Kim, J.-G., Hembram, K. P. S. S., Kim, Y.-I., Min, B.-K., Park, Y., Lee, J.-K., Moon, D. J., Lee, W., Lee, S.-G. \& John, P. (2016). Sci. Rep. 6, 39624.

Lee, J.-K., Lee, S.-C., Ahn, J.-P., Kim, S.-C., Wilson, J. I. B. \& John, P. (2008). J. Chem. Phys. 129, 234709.
Li, J., Hu, Z., Yi, Y., Yu, M., Li, X., Zhou, J., Yin, J., Wu, S. \& Guo, W. (2019). Small, 15, 1805188.

Li, L. H., Chen, Y., Behan, G., Zhang, H., Petravic, M. \& Glushenkov, A. M. (2011). J. Mater. Chem. 21, 11862-11866.

Liu, L., Feng, Y. P. \& Shen, Z. X. (2003). Phys. Rev. B, 68, 104102.

Matović, B., Luković, J., Nikolić, M., Babić, B., Stanković, N., Jokić, B. \& Jelenković, B. (2016). Ceram. Int. 42, 16655-16658.

Methfessel, M. P. \& Paxton, A. T. (1989). Phys. Rev. B, 40, 36163621.

Monkhorst, H. J. \& Pack, J. D. (1976). Phys. Rev. B, 13, 5188-5192.

Novoselov, K. S., Geim, A. K., Morozov, S. V., Jiang, D., Zhang, Y., Dubonos, S. V., Grigorieva, I. V. \& Firsov, A. A. (2004). Science, 306, 666-669.

Palmer, D. C. (2014). CrystalMaker. CrystalMaker Software Ltd, Begbroke, Oxfordshire, England. http://crystalmaker.com/.

Pease, R. S. (1950). Nature, 165, 722-723.

Pease, R. S. (1952). Acta Cryst. 5, 356-361.

Perdew, J. P., Burke, K. \& Ernzerhof, M. (1996). Phys. Rev. Lett. 77, 3865-3868.

Pierson, H. O. (1993). In Handbook of Carbon, Graphite, Diamond and Fullerenes. New Jersey, USA: Noyes Publications.

Ribeiro, M. \& Peres, M. R. (2011). Phys. Rev. B, 83, 235312.

Rodríguez-Carvajal, J. (2001). Newslett. Powder Diff. IUCr, 26, 12-19.

Sutter, P., Lahiri, J., Zahl, P., Wang, B. \& Sutter, E. (2013). Nano Lett. 13, 276-281.

Tonkikh, A. A., Voloshina, E. N., Werner, P., Blumtritt, H., Senkovskiy, B., Güntherodt, G., Parkin, S. S. P. \& Dedkov, Y. S. (2016). Sci. Rep. 6, 23547.

Topsakal, M., Aktürk, E. \& Ciraci, S. (2009). Phys. Rev. B, 79, 115442.

Vanderbilt, D. (1990). Phys. Rev. B, 41, 7892-7895.

Wang, J., Ma, F. \& Sun, M. (2017). RSC Adv. 7, 16801-16822.

Warner, J. H., Rümmeli, M. H., Bachmatiuk, A. \& Büchner, B. (2010). ACS Nano, 4, 1299-1304.

Yuan, S., Toury, B., Journet, C. \& Brioude, A. (2014). Nanoscale, 6, 7838-7841.

Zhang, K., Feng, Y., Wang, F., Yang, Z. \& Wang, J. (2017). J. Mater. Chem. C, 5, 11992-12022. 